\title{
Downregulation of miR-129-2 by promoter hypermethylation regulates breast cancer cell proliferation and apoptosis
}

\author{
XIAOFENG TANG $^{1^{*}}$, JIANJUN TANG $^{2 *}$, XIA LIU $^{4}$, LEI ZENG $^{3}$, CHUN CHENG $^{1}$, YANQIN LUO $^{1}$, LIPING LI $^{1}$, \\ SHU-LAN QIN ${ }^{1}$, YI SANG ${ }^{1}$, LIANG-MING DENG ${ }^{5}$ and XIAO-BIN LV ${ }^{1}$ \\ ${ }^{1}$ Nanchang Key Laboratory of Cancer Pathogenesis and Translational Research, Center Laboratory, \\ The Third Affiliated Hospital, Nanchang University, Nanchang, Jiangxi 330008; ${ }^{2}$ Department of Gastroenterology, \\ Jiangxi Cancer Hospital, Nanchang, Jiangxi 330029; ${ }^{3}$ Department of Radiation Oncology, Jiangxi Cancer Hospital, \\ Nanchang, Jiangxi 330029; ${ }^{4}$ Department of Oncology, Guangzhou First Municipal People's Hospital Affiliated \\ to Guangzhou Medical College, Guangzhou, Guangdong 510180; ${ }^{5}$ Department of Medicine, \\ Gaoming Heshui Hospital, Foshan, Guangdong 528500, P.R. China
}

Received November 15, 2015; Accepted December 29, 2015

DOI: 10.3892/or.2016.4647

\begin{abstract}
Aberrant expression of the miR-129 family has been found in several types of cancer, yet its expression and potential biologic role in breast cancer remain largely unknown. In the present study, we found that miR-129-2 was consistently downregulated in the breast cancer specimens and cell lines. Overexpression of miR-129-2-3p markedly suppressed breast cancer cell proliferation and induced its apoptosis. In addition, a luciferase reporter assay revealed that miR-129-2-3p suppressed BCL2L2 expression. Furthermore, BCL2L2 was able to reverse miR-129-2-3p-mediated cell apoptosis, indicating that BCL2L2 plays a crucial role in mediating the tumor-suppressive role of miR-129-2-3p. Moreover, bisulfite DNA sequencing PCR (BSP) analysis identified that promoter hypermethylation was responsible for the downregulation of miR-129-2 in breast cancer. Collectively, our findings indicate that miR-129-2 is downregulated in breast cancer cells by promoter hypermethylation. Moreover, downregulation of miR-129-2 results in BCL2L2 overexpression and disease progression in breast cancer patients.
\end{abstract}

Correspondence to: Professor Xiao-Bin Lv, Nanchang Key Laboratory of Cancer Pathogenesis and Translational Research, Center Laboratory, The Third Affiliated Hospital, Nanchang University, Nanchang, Jiangxi 330008, P.R. China

E-mail: promab2006@126.com

Dr Liang-Ming Deng, Department of Medicine, Gaoming Heshui Hospital, Foshan, Guangdong, P.R. China

E-mail: 2806119774@qq.com

${ }^{*}$ Contributed equally

Key words: miR-129-2, methylation, BCL2L2, breast cancer, apoptosis, proliferation

\section{Introduction}

Breast cancer is the most common cause of cancer-related mortality among women in developed and developing countries (1). In 2013 in the United States, 232,340 women were diagnosed with invasive breast cancer, and 39,620 succumbed to the disease (2). The exploration of the underlying mechanisms involved in breast cancer have been the subject of extensive research over the past decades. However, the mechanisms involved in breast cancer tumorigenesis and progression remain poorly understood.

MicroRNAs (miRNAs) are small, conserved, non-coding RNA sequences that suppress mRNA translation and/or degrade mRNA molecules by binding to their 3'-untranslated regions. Differential expression of miRNAs has been widely described in breast cancer tissue compared to normal tissue suggesting that miRNAs may function as oncogenes or tumorsuppressors (3-5).

The miR-129 family is one of the typical cancer-associated miRNAs. It has two precursors, miR-129-1 and miR-129-2. Two mature miRNAs are processed from the 5'-prime and 3'-prime of its pre-miRNA precursor. For miR-129-1 and miR-129-2, their 5'-prime product, miR-129-5p, is the same. However, their 3'-prime products are different, which are named miR129-1-3p and miR-129-2-3p, respectively $(6,7)$. Dysregulation of miR-129-2 is frequently detected in solid tumors (8-13). However, the role of miR-129-2 in breast cancer remains unknown.

The methylation of $\mathrm{CpG}$ islands in gene promoters has been strongly linked to the silencing of the expression of tumor-suppressor genes in different types of cancers (14-17). The hypermethylation of promoter $\mathrm{CpG}$ islands has been found to affect not only tumor-suppressor mRNAs, but also tumor-suppressor miRNAs. Several tumor-associated miRNAs have been reported to be silenced by the aberrant hypermethylation of their promoter regions in breast cancer, including miR-124, miR-34c, miR-148a, miR-155, miR-203 and miR-129 (3,18-22).

In the present study, we found that the miR-129-2 promoter was hypermethylated in breast cancer cells. Furthermore, we found 
that miR-129-2-3p functions as a tumor suppressor by inducing the apoptosis of breast cancer cells by targeting BCL2L2.

\section{Materials and methods}

Cell lines and culture. Human breast cancer cell lines (MCF-7, T47D, HMLER, and MDA-MB-231) were cultured in Dulbecco's modified Eagle's medium (DMEM; Life Technologies) supplemented with $10 \%$ fetal bovine serum [Biological Industries (BI)]. Immortalized breast epithelial cell lines (MCF-10A and 76N) were cultured in DMEM/F-12 1:1 mix supplemented with human insulin $(10 \mu \mathrm{g} / \mathrm{ml})$, epidermal growth factor $(20 \mathrm{ng} / \mathrm{ml})$, cholera toxin $(50 \mu \mathrm{l})$, hydrocortisone $(0.5 \mu \mathrm{g} / \mathrm{ml})$, and horse serum $(5 \%)$. All cell lines were incubated in a humidified chamber with $5 \% \mathrm{CO}_{2}$ at $37^{\circ} \mathrm{C}$.

Fifteen pairs of fresh primary breast cancer tissues and non-cancerous tissues were obtained at the time of diagnosis prior to any therapy from the Cancer Hospital of Jiangxi Province (Nanchang, China). The clinical processes were approved by the Ethics Committees of Nanchang University and informed consent was collected from each patient.

Oligonucleotide transfection. The miR-129-2-5p and miR-1292-3p mimics and a non-specific miRNA control (NC) were synthesized by GenePharma (Shanghai, China). miRNAs were transfected at a working concentration of $50 \mathrm{nmol} / \mathrm{l}$ using RNAiMAX reagent (Invitrogen, USA) according to the manufacturer's instructions. The transfected cells were incubated at $37^{\circ} \mathrm{C}$ for $24 \mathrm{~h}$ in complete medium and harvested at the indicated time-points.

RNA extraction, reverse transcription, and quantitative real-time PCR. Total RNA was extracted using TRIzol (Invitrogen) according to the manufacturer's instructions. cDNA was synthesized with the MLV transcriptase kit (Invitrogen). The quantitative analysis of miR-129-2-3p expression was assayed using a Bulge-Loop ${ }^{\mathrm{TM}}$ miRNA qRT-PCR primer (RiboBio, Guangzhou, China) and Platinum ${ }^{\circledR} \mathrm{SYBR}^{\circledR}$ Green qPCR SuperMix-UDG with ROX (Invitrogen) on an ABI 7900HT instrument (Applied Biosystems, Foster, CA, USA) according to the manufacturer's instructions, and U6 small nuclear RNA (U6-snRNA) purchased from RiboBio was used as an internal control. The fold changes were calculated through relative quantification with $2^{-\Delta \Delta \mathrm{Ct}}$. All of the reactions were performed in a $20-\mu 1$ reaction volume in triplicate.

Western blot analysis. This procedure was detailed previously (23). Briefly, protein lysates were resolved through $10 \%$ SDS-PAGE and electrophoretically transferred to a PVDF (polyvinylidene difluoride) membrane (Millipore, USA). Then, the membrane was probed with an antibody against human BCL2L2 (1:1,000 dilution; Cell Signaling Technology) or $\beta$-actin (Bioworld, USA) as a protein loading control, followed by peroxidase-conjugated goat anti-mouse $\operatorname{IgG}(\mathrm{H}+\mathrm{L}$; Proteintech, China) as the secondary antibody. The intensity of the protein fragments was visualized with an X-ray image film processing machine (Kodak, Japan).

MTT and colony formation assays. For cell proliferation assays, cells transfected with miRNAs or co-transfected with
miRNAs and plasmids for $24 \mathrm{~h}$ were reseeded in 96-well plates at $1.5 \times 10^{3}$ cells/well in a final volume of $150 \mu \mathrm{l}$ and incubated overnight. The effect of miR-129-2-3p/BCL2L2 on cell growth and proliferation was determined with an MTT assay as described previously (24).

For the colony formation assays, after $24 \mathrm{~h}$ of transfection, the cells were reseeded in 6-well plates at 1,000 cells/well, and the medium was replaced every 3 days. After incubation at $37^{\circ} \mathrm{C}$ for 2 weeks, the cells were washed twice with PBS, fixed and stained with $0.5 \%$ crystal violet. The number of colonies was counted under a microscope.

Cell cycle profile assay. A cell cycle profile assay was performed as described previously (25). Briefly, the cells were harvested by trypsinization and collected by centrifugation. The cells were washed twice with phosphate-buffered saline (PBS) and fixed in $1 \mathrm{ml}$ of $70 \%$ ethanol at $4^{\circ} \mathrm{C}$ for $1 \mathrm{~h}$ to overnight. The cells were washed twice with $\mathrm{PBS} / 1 \%$ bovine serum albumin (BSA) and then incubated with $1 \mathrm{ml}$ of PBS/1\% BSA containing $30 \mathrm{mg} /$ $\mathrm{ml}$ propidium iodide (PI) and $0.25 \mathrm{mg} / \mathrm{ml} \mathrm{RNase}$ A for $30 \mathrm{~min}$ at room temperature. The cells were then analyzed for DNA content by flow cytometry using a cytomics FACSCalibur (BD Biosciences, USA). The data were analyzed using Modifit.

Apoptosis assay. Apoptosis was tested using Annexin V and PI double staining kits according to the manufactory's instructions. Briefly, MDA-MB-231 and MCF-7 cells were transfected with miR-129-2-3p or the control for $48 \mathrm{~h}$. The cells were then harvested and washed twice with PBS. Then the cells were incubated with Annexin V and PI for $30 \mathrm{~min}$. Cells were analyzed by flow cytometry using a cytomics FACSCalibur (BD Biosciences).

Luciferase reporter assay. The 3'UTR sequence of BCL2L2 containing the putative miR-129-2-3p binding site was amplified with PCR and cloned into the pGL3-control vector (Promega, Madison, WI, USA) downstream of the firefly luciferase gene, which was designated as wild-type 3'UTR (wt 3'UTR). Mutagenesis was performed using a Quik-Change Site-Directed Mutagenesis kit (Stratagene, USA) according to the manufacturer's instructions, resulting in mutated 3'UTR (mut 3'UTR). The primers used for the construction of luciferase reporters were BCL2L2 wt (forward, AATCTAGAA CCCTGCCTGTGGTCCTGA and reverse, AACTCTAGA AGAGAGTCCCTAGT). The Wt or mut 3'UTR vector and the control vector pRL-TK (Promega) coding for Renilla luciferase were co-transfected with miR-129-2-3p mimics or NC into the MDA-MB-231 cells using Lipofectamine 2000 (Invitrogen). The luciferase activity was measured $48 \mathrm{~h}$ later using the Dual-Luciferase Reporter Assay system (Promega). The firefly luciferase values were normalized to Renilla, and the ratio of firefly/Renilla values is presented. The experiments were performed independently in triplicate.

Rescue experiments. The open reading frame (ORF) encoding BCL2L2 protein was amplified and cloned into pcDNA3.1 with the Myc tag located in the N-terminal of BCL2L2. The primers used for amplified BCL2L2 were forward, AAGGTA CCACCATGGCGACCCCAGCCTCG and reverse, AACTCG AGTCACTTGCTAGCAAAAAAGGC. Breast cancer cells co-transfected with miR-129-2-3p mimics and the BCL2L2- 

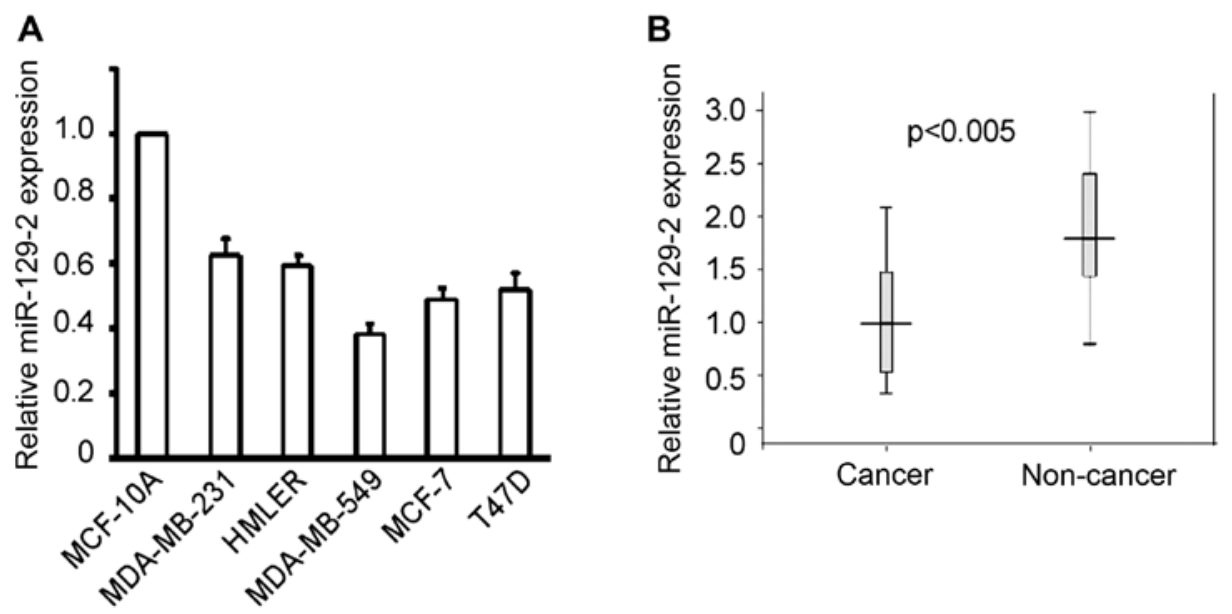

Figure 1. miR-129-2 is dysregulated in breast cancer. (A) The miR-129-2 level was reduced in breast cancer cells compared to the level in immortalized epithelial cells. (B) The miR-129-2 level was evaluated in 24 pairs of freshly frozen primary breast cancer tissues and breast cyst tissues using qRT-PCR.

expressing vector or empty plasmid for $24 \mathrm{~h}$ were trypsinized and subjected to apoptosis assay.

Genomic DNA isolation and bisulfite DNA sequencing PCR (BSP) analysis. Genomic DNAs were isolated from the cells using the DNeasy Tissue kit (Qiagen, USA). DNA samples were treated with sodium bisulfite to convert cytosine to uracil using the Methyl Detector ${ }^{\mathrm{TM}}$ Bisulfite Modification kit (Active Motif, North America) according to the manufacturer's instructions. For BSP, a 1- $\mu 1$ aliquot of sodium bisulfite-treated DNA was amplified by PCR with commonly used primers for methylated and unmethylated DNA sequences. The PCR products were cloned into the pGEMT Easy vector (Promega), and 10 clones from each sample were sequenced to determine the methylation status of each CpG site. BSP primers for miR-129-2 were forward, TAGGGATTTGAAGATAGTGTTTTTAT and reverse, AAAAAAACCTCACCCAAAATAAATTA, and were designed according to previously validated oligonucleotides and synthesized commercially (Invitrogen).

Statistical analysis. Statistical analyses were performed using SPSS 16.0 software (SPSS Inc., Chicago, IL, USA). The values are presented as the mean \pm standard deviation (SD) of three independent experiments. Differences between two groups were evaluated by a two-tailed Student's t-test. The relationship between BCL2L2 and miR-129-2-3p expression was assessed with two-tailed Pearson's correlation. Differences were considered to be statistically significant at $\mathrm{P}<0.05$ and $\mathrm{P}<0.01$ as indicated in the figure legends.

\section{Results}

miR-129-2 is downregulated in breast cancer cells as well as in clinical samples. To explore the potential role of miR-129-2 in breast cancer progression, we examined the miR-129-2 level in breast cancer cells and tissues. Since the sequence of miR-129-1-5p and miR-129-2-5p are the same, we evaluated the miR-129-2 level using primers specific to miR-129-2-3p. As shown in Fig. 1A, the level of miR-129-2-3p detected in the breast cancer cells was significantly lower than that in immor- talized breast cells. In addition, the level of miR-129-2-3p was also downregulated in the breast cancer tissues compared with normal breast tissues (Fig. 1B). These results suggest that miR-129-2-3p may exert a tumor-suppressor function in breast cancer.

miR-129-2-3p suppresses the cell growth and colony formation of breast cancer cells. The above results led to us to investigate the functional impact of miR-129-2 on breast cancer cells. We firstly transfected miR-129-5p into MDA-MB-231 cells and found that it did not affect the proliferation of MDA-MB-231 cells (data not shown). We next investigated the effect of miR$129-2-3 p$ on the breast cancer cells. The efficient transfection of miR-129-2-3p mimics into the breast cancer cells was validated by qRT-PCR (Fig. 2A). Notably, we observed a major reduction in the cell growth of the MDA-MB-231 cells upon miR-129-2-3p transfection compared with the cell growth noted in the control (Fig. 2B). Accordingly, similar results were also observed in the MCF-7 cells (Fig. 2C). Furthermore, consistent with the proliferation assay, transfection of miR129-2-3p mimics markedly suppressed the colony formation of both the MDA-MB-231 (Fig. 2D) and MCF-7 cells (Fig. 2E).

miR-129-2-3p induces the apoptosis of breast cancer cells. Give that the miR-129-2-3p mimic transfection suppressed the cell growth of breast cancer cells, we sought to explore its underlying mechanisms. We first detected the effect of miR-129-2-3p mimics on the apoptosis of breast cancer cells. As shown in Fig. 3A, miR129-2-3p mimic transfection led to a notably elevated apoptotic rate in the MDA-MB-231 cells compared to the apoptotic rate noted in the $\mathrm{NC}$ control. Consistently, this similar result was also observed in the MCF-7 cells (Fig. 3B). However, transfection of the miR-129-2-3p mimics only had a slight influence on the cell cycle profile of both the MDA-MB-231 (Fig. 3C) and MCF-7 cells (Fig. 3D). Taken together, these results indicate that miR129-2-3p functions as a tumor suppressor of breast cancer mainly through induction of apoptosis.

BCL2L2 is a direct target of miR-129-2-3p in breast cancer cells. We used the online bioinformatic prediction algorithm 

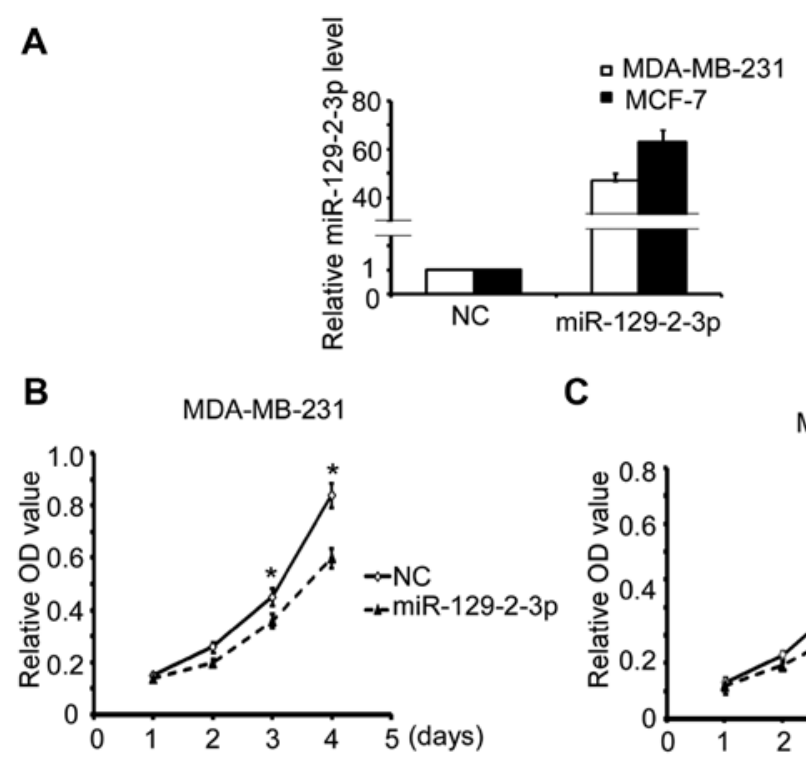

C

D
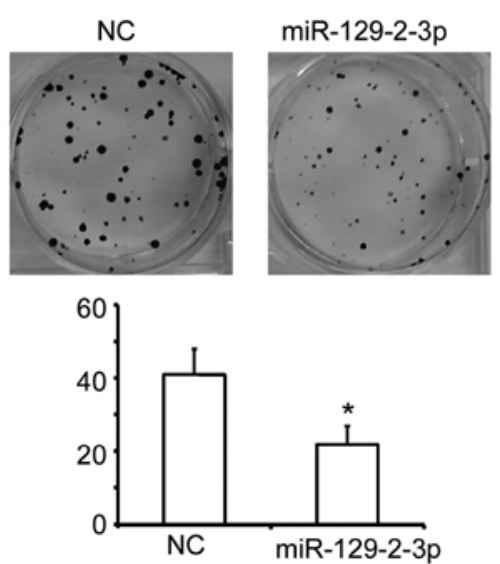

MCF-7

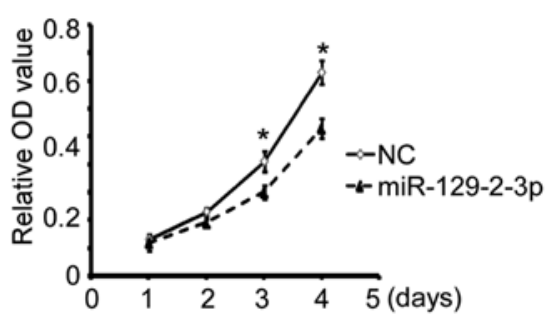

E
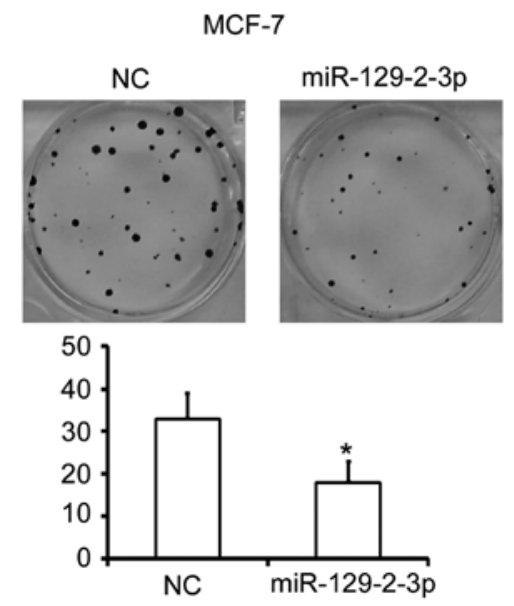

Figure 2. Overexpression of miR-129-2-3p suppresses the proliferation and colony formation of breast cancer MDA-MB-231 and MCF-7 cells. (A) The efficient transfection of miR-129-2-3p into the MDA-MB-231 and MCF-7 cells was evaluated by qRT-PCR. (B and C) The proliferation of MDA-MB-231 and MCF-7 cells transfected with miR-129-2-3p were assessed by MTT assay. " $\mathrm{P}<0.05$ compared with the control. (D and E) Overexpression of miR-129-2-3p suppresses the colony formation of MDA-MB-231 and MCF-7 cells. " $\mathrm{P}<0.05$ compared with the control.

(http://ferrolab.dmi.unict.it/miro/) to analyze the direct mRNA targets of miR-129-2-3p. Of all the hypothetical targets of miR-129-2-3p, BCL2L2, an oncogene with a 3'UTR containing the miR-129-2-3p targeting site provoked our interest (Fig. 4A). The dual-luciferase reporter assay showed that miR-129-2-3p mimics reduced the fluorescence activity of wt 3'UTR by $50 \%$ compared with the negative control (Fig. 4B, $\mathrm{P}<0.01$ ), whereas mut 3 'UTR showed an abrogated response to miR-129-2-3p (Fig. 4B). In addition, miR-129-2-3p mimic transfection reduced the protein level of BCL2L2 in the MDA-MB-231 cells (Fig. 4C). More importantly, reintroduction of BCL2L2 reversed the miR-129-2-3p mimic transfection-induced apoptosis of the breast cancer cells (Fig. 4D). Taken together, these results indicate that BCL2L2 is a direct target of miR-129-2-3p and is responsible for the miR-129-2-3p-induced apoptosis of breast cancer cells. is attributed in part to promoter DNA hypermethylation.
To explore the potential mechanisms involved in the downregulation of miR-129-2 in breast cancer cells, we analyzed the methylation status of the $\mathrm{CpG}$ island of the miR-129-2 promoter using BSP sequencing. As shown in Fig. 5A, the promoter of miR-129-2 was heavily methylated in the MDA-MB-231 and T47D breast cancer cells but slightly methylated in the immortalized breast $76 \mathrm{~N}$ cells (Fig. 5A). More importantly, treatment of MDA-MB-231 and T47D cells with de-methylation reagent 5-Aza-dC increased the miR-129-2 level (Fig. 5B). Taken together, these results indicate that miR-129-2 expression is regulated by promoter hypermethylation.

\section{Discussion}

In recent years, growing evidence has revealed that miRNAs play important roles in tumor development and progression. While in different tumor types tissue-specific differences exist, diverse molecular pathways in miRNA signaling and regulation are also important factors to consider when 
A
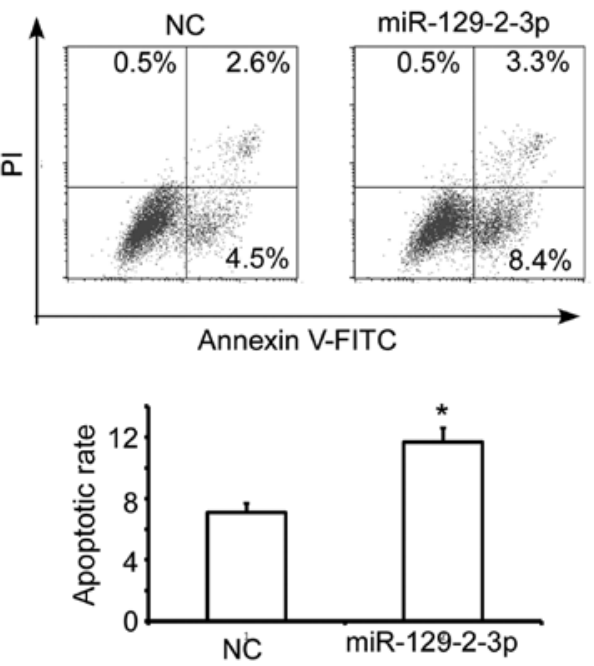

C
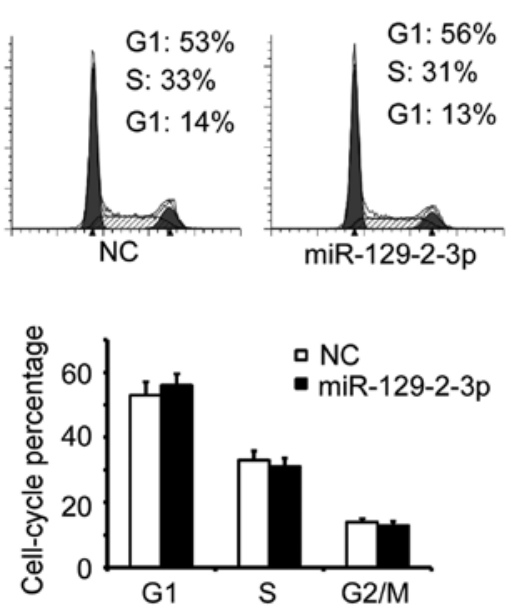

B
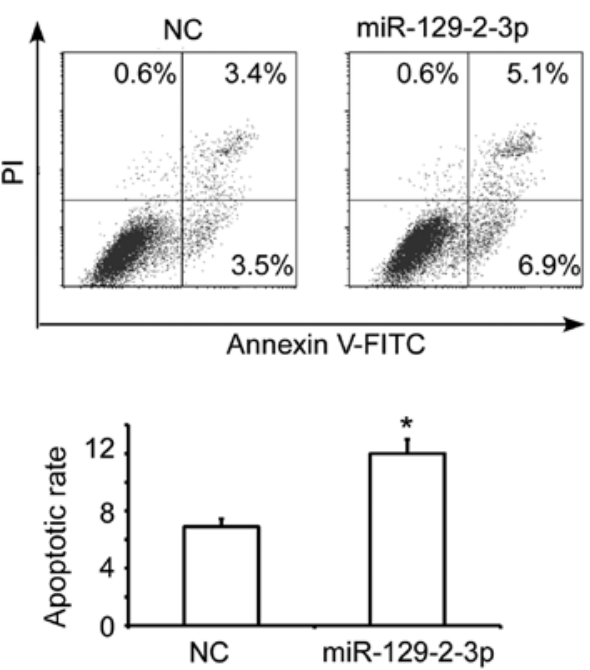

D
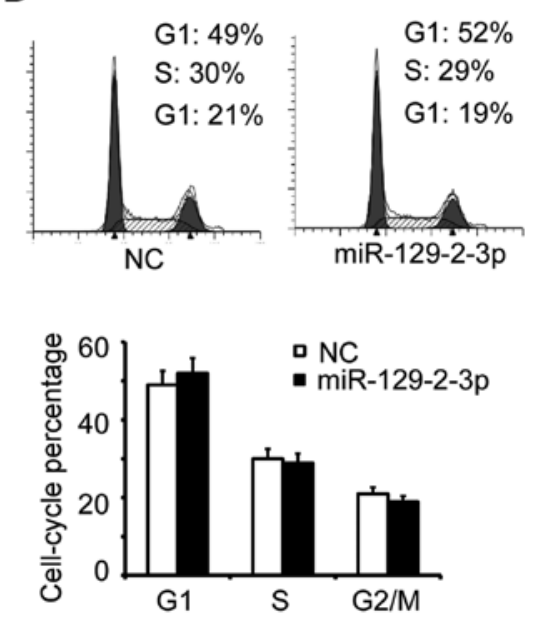

Figure 3. Overexpression of miR-129-2-3p induces the apoptosis of breast cancer cells. MDA-MB-231 (A) and MCF-7 cells (B) were transiently transfected with miR-129-2-3p mimics or miRNA control (NC) for $48 \mathrm{~h}$ and the apoptosis rate was examined by flow cytometry. ${ }^{*} \mathrm{P}<0.05$ compared with the control. (C and D) MDA-MB-231 and MCF-7 cells were transiently transfected with miR-129-2-3p mimics or miRNA control (NC) for $48 \mathrm{~h}$ and the cell cycle profiles were examined by flow cytometry.

approaching the discrepancies in mRNA expression in cancer. Therefore, it is necessary to profile the molecular function and the underlying mechanisms of cancer-related miRNAs in each tumor type. Few studies have investigated the roles of the miR-129 family members in cell proliferation and apoptosis in breast cancer. Here, we demonstrated that miR-129-2 was downregulated in human breast cancer cells and clinical samples. Functional studies demonstrated that overexpression of miR-129-2-3p suppressed the cell growth, colony formation and induced apoptosis of the breast cancer cells. In addition, prediction and a luciferase reporter assay revealed that miR129-2-3p directly targets BCL2L2 and inhibits its expression. Furthermore, BSP sequence results showed that miR-129-2 was heavily methylated in the breast cancer cells and tissues. 5'-Aza-dC treatment increased the expression of miR-129-2-3p in the MDA-MB-231 and T47D cells, indicating that promoter hypermethylation is an important factor contributing to the downregulation of miR-129-2-3p in breast cancer.

The miR-129 family includes two precursors miR-129-1 and miR-129-2 which are processed to three mature miRNAs,
miR-129-5p, miR-129-1-3p and miR-129-2 (7). Dual functions for miR-129, as a tumor suppressor and oncogene, have been observed in different types of cancers. It has been reported that miR-129-5p is downregulated in neuroendocrine tumors, gastric cancer, ovarian cancer and medullary thyroid carcinoma and plays a tumor-suppressor role in these tumors (22,26-28). Epigenetic regulation of miR-129-2 was reported in glioma and lung cancer $(8,9)$. In contrast, however, miR-129 is also upregulated in several solid tumors and non-cancerous tissues from cancer patients with lymph node metastases $(29,30)$. Our results revealed the tumor-suppressor functions of miR-129-2.miRNAs degrade or repress translation of target mRNAs. Therefore, to validate the oncogenic function of miR-129-2, an oncogenic target was required to be identified. We predicted several potential target candidates for miR-129-2-3p. Among them, BCL2L2 provoked our interesting due to its oncogenic role in many solid tumors (31-33). Luciferase report assay confirmed that miR129-2-3p directly targeted BCL2L2 3'UTR for degradation. More importantly, rescue assay results indicated that BCL2L2 is crucial for miR-129-2-3p-induced breast cancer apoptosis. 
A

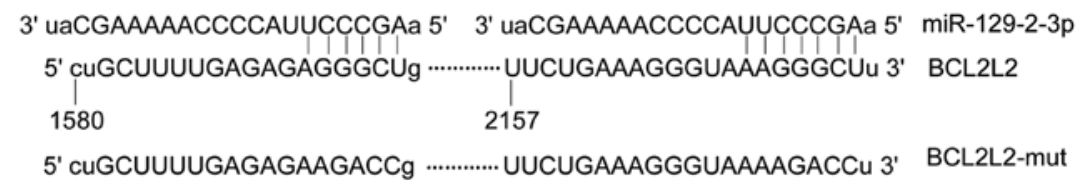

B
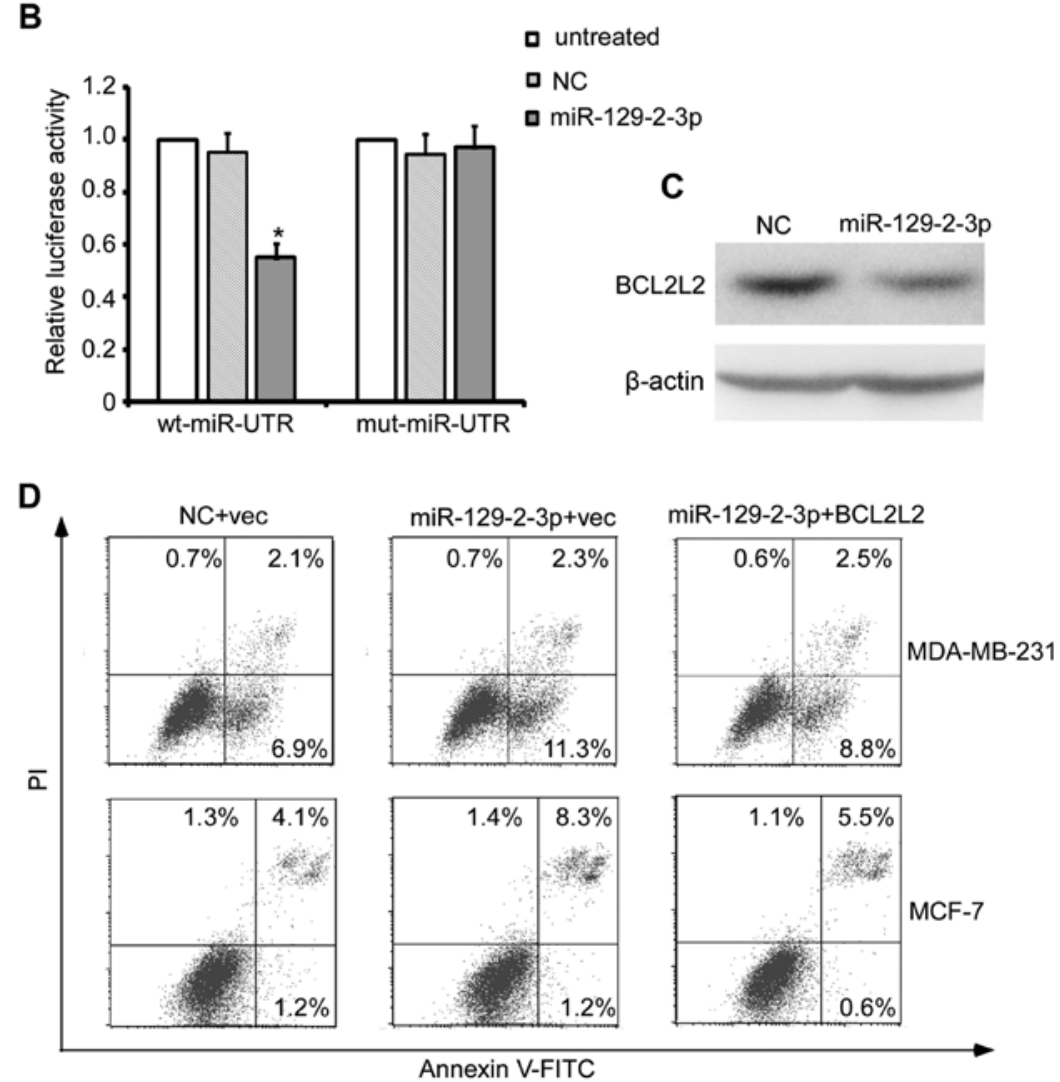

Figure 4. BCL2L2 is a direct target of miR-129-2-3p in breast cancer cells. (A) Schematic diagram of the reporter constructs containing the predicted miR-1292-3p binding site in the 3 'UTR of BCL2L2. (B) The overexpression of miR-129-2-3p using the miRNA mimics significantly attenuated the luciferase activity of the BCL2L2 3'UTR in the MDA-MB-231 cells. The co-transfection of wt or mut 3'UTR and miRNAs is indicated. "P $<0.05$ compared with the control. (C) The overexpression of miR-129-2-3p reduced the BCL2L2 protein levels. (D) Overexpression of BML2L2 counteracts miR-129-2-3p mimic transfection-induced apoptosis in the breast cancer cells.

A

MDA-MB231

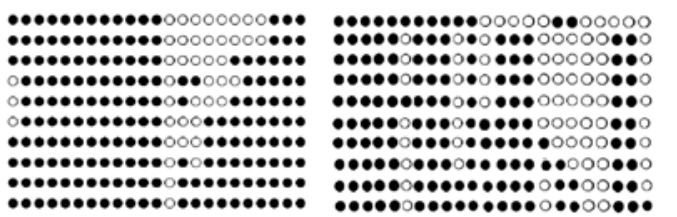

B

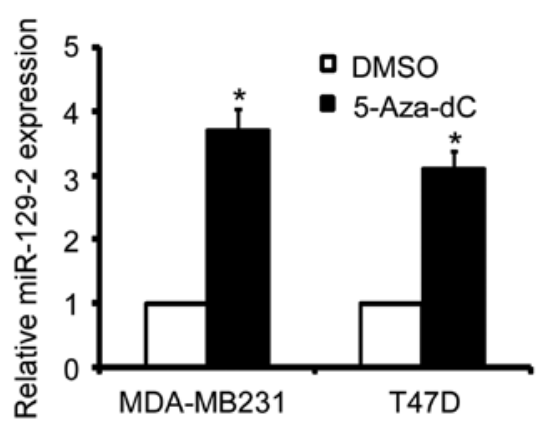

Figure 5. Promoter hypermethylation reduces miR-129-2 expression. (A) The methylation status of the CpG in the miR-129-2 promoter was analyzed by bisulfite sequencing. Black circle, methylated $\mathrm{CpG}$ site; white circle, unmethylated $\mathrm{CpG}$ site. (B) 5-Aza-dC treatment increased the miR-129-2 level. " $\mathrm{P}<0.05$ compared with the control. 
DNA hypermethylation is closely associated with gene inactivation and is a common and widespread phenomenon in human tumors. Many previous studies have reported that promoter hypermethylation-induced downregulation of tumor-suppressor miRNAs correlates with carcinogenesis. Our data revealed that the hypermethylation of the miR-129-2 promoter led to the downregulation of the miR-129-2 level in breast cancer patients. In addition, demethylation agent 5-Aza-dC treatment increased miR-129-2 expression in the breast cancer cells. These results indicate that promoter hypermethylation leads to the downregulation of miR-129-2-3p in breast cancer, at least in part.

In conclusion, our study suggests that miR-129-2 is downregulated by DNA methylation and plays a tumor-suppressor role in breast cancer by targeting BCL2L2 for degradation. Transfection of miR-129-2-3p was found to suppress cell growth and promote the cell apoptosis of breast cancer cells, which may provide a novel therapeutic strategy for the treatment of breast cancer.

\section{Acknowledgements}

The present study was supported by grants from the Natural Science Foundation of China (81560452 and 81102023 to X.B.L.; 81560403 to J.T.).

\section{References}

1. Torre LA, Bray F, Siegel RL, Ferlay J, Lortet-Tieulent J and Jemal A: Global cancer statistics, 2012. CA Cancer J Clin 65: 87-108, 2015

2. DeSantis C, Ma J, Bryan L and Jemal A: Breast cancer statistics, 2013. CA Cancer J Clin 64: 52-62, 2014.

3. Diao Y, Guo X, Jiang L, Wang G, Zhang C, Wan J, Jin Y and Wu Z: miR-203, a tumor suppressor frequently down-regulated by promoter hypermethylation in rhabdomyosarcoma. J Biol Chem 289: 529-539, 2014.

4. Gong C, Qu S, Lv XB, Liu B, Tan W, Nie Y, Su F, Liu Q, Yao H and Song E: BRMS1L suppresses breast cancer metastasis by inducing epigenetic silence of FZD10. Nat Commun 5: 5406, 2014.

5. Lu K, Wang J, Song Y, Zhao S, Liu H, Tang D, Pan B, Zhao H and Zhang Q: miRNA-24-3p promotes cell proliferation and inhibits apoptosis in human breast cancer by targeting $\mathrm{p} 27^{\mathrm{Kip} 1}$. Oncol Rep 34: 995-1002, 2015.

6. Cui L, Zhang X, Ye G, Zheng T, Song H, Deng H, Xiao B, $\mathrm{Xia} \mathrm{T}, \mathrm{Yu} \mathrm{X}$, Le Y, et al: Gastric juice microRNAs as potential biomarkers for the screening of gastric cancer. Cancer 119: 1618-1626, 2013

7. Katada T, Ishiguro H, Kuwabara Y, Kimura M, Mitui A, Mori Y, Ogawa R, Harata K and Fujii Y: microRNA expression profile in undifferentiated gastric cancer. Int J Oncol 34: 537-542, 2009.

8. Tian XY, Zhang L, Sun LG and Li M: Epigenetic regulation of miR-129-2 leads to overexpression of PDGFRa and FoxP1 in glioma cells. Asian Pac J Cancer Prev 16: 6129-6133, 2015.

9. Xiao Y, Li X, Wang H, Wen R, He J and Tang J: Epigenetic regulation of miR-129-2 and its effects on the proliferation and invasion in lung cancer cells. J Cell Mol Med 19: 2172-2180, 2015

10. Yang Y, Huang JQ, Zhang X and Shen LF: miR-129-2 functions as a tumor suppressor in glioma cells by targeting HMGB1 and is down-regulated by DNA methylation. Mol Cell Biochem 404: 229-239, 2015

11. He Y, Huang C, Zhang L and Li J: Epigenetic repression of miR-129-2 in cancer. Liver Int 34: 646, 2014.

12. Kang M, Li Y, Liu W, Wang R, Tang A, Hao H, Liu Z and Ou H: miR-129-2 suppresses proliferation and migration of esophageal carcinoma cells through downregulation of SOX4 expression. Int J Mol Med 32: 51-58, 2013.

13. Lu CY, Lin KY, Tien MT, Wu CT, Uen YH and Tseng TL: Frequent DNA methylation of miR-129-2 and its potential clinical implication in hepatocellular carcinoma. Genes Chromosomes Cancer 52: 636-643, 2013.
14. Bedi U, Mishra VK, Wasilewski D, Scheel C and Johnsen SA: Epigenetic plasticity: A central regulator of epithelial-to-mesenchymal transition in cancer. Oncotarget 5: 2016-2029, 2014.

15. Chen QW, Zhu XY, Li YY and Meng ZQ: Epigenetic regulation and cancer (Review). Oncol Rep 31: 523-532, 2014.

16. Sang Y, Cheng C, Tang XF, Zhang MF and Lv XB: Hypermethylation of TET1 promoter is a new diagnosic marker for breast cancer metastasis. Asian Pac J Cancer Prev 16: 1197-1200, 2015.

17. Dong Y and Wang A: Aberrant DNA methylation in hepatocellular carcinoma tumor suppression (Review). Oncol Lett 8: 963-968, 2014.

18. Lv XB, Jiao Y, Qing Y, Hu H, Cui X, Lin T, Song E and Yu F: miR-124 suppresses multiple steps of breast cancer metastasis by targeting a cohort of pro-metastatic genes in vitro. Chin J Cancer 30: 821-830, 2011.

19. Yu F, Jiao Y, Zhu Y, Wang Y, Zhu J, Cui X, Liu Y, He Y, Park EY, Zhang $\mathrm{H}$, et al: MicroRNA 34c gene down-regulation via DNA methylation promotes self-renewal and epithelial-mesenchymal transition in breast tumor-initiating cells. J Biol Chem 287: 465-473, 2012.

20. Li HP, Huang HY, Lai YR, Huang JX, Chang KP, Hsueh C and Chang YS: Silencing of miRNA-148a by hypermethylation activates the integrin-mediated signaling pathway in nasopharyngeal carcinoma. Oncotarget 5: 7610-7624, 2014

21. Yim RL, Wong KY, Kwong YL, Loong F, Leung CY, Chu R, Lam WW, Hui PK, Lai R and Chim CS: Methylation of miR-155-3p in mantle cell lymphoma and other non-Hodgkin's lymphomas. Oncotarget 5: 9770-9782, 2014.

22. Wu Q, Yang Z, Xia L, Nie Y, Wu K, Shi Y and Fan D: Methylation of miR-129-5p CpG island modulates multi-drug resistance in gastric cancer by targeting $\mathrm{ABC}$ transporters. Oncotarget 5: $11552-11563,2014$

23. Lv XB, Wu W, Tang X, Wu Y, Zhu Y, Liu Y, Cui X, Chu J, $\mathrm{Hu} \mathrm{P}, \mathrm{Li}$ J, et al: Regulation of SOX10 stability via ubiquitination-mediated degradation by Fbxw $7 \alpha$ modulates melanoma cell migration. Oncotarget 6: 36370-36382, 2015.

24. Lv XB, Zhang X, Deng L, Jiang L, Meng W, Lu Z and Wang X: miR-92a mediates AZD6244 induced apoptosis and G1-phase arrest of lymphoma cells by targeting Bim. Cell Biol Int 38: 435-443, 2014.

25. Salilew-Wondim D, Hölker M, Rings F, Phatsara C, Mohammadi-Sangcheshmeh A, Tholen E, Schellander K and Tesfaye D: Depletion of BIRC6 leads to retarded bovine early embryonic development and blastocyst formation in vitro. Reprod Fertil Dev 22: 564-579, 2010.

26. Døssing KB, Binderup T, Kaczkowski B, Jacobsen A, Rossing M, Winther O, Federspiel B, Knigge U, Kjær A and Friis-Hansen L: Down-regulation of miR-129-5p and the let-7 family in neuroendocrine tumors and metastases leads to up-regulation of their targets Egr1, G3bp1, Hmga2 and Bach1. Genes (Basel) 6: 1-21, 2014.

27. Tan G, Cao X, Dai Q, Zhang B, Huang J, Xiong S, Zhang Y, Chen W, Yang J and Li H: A novel role for microRNA-129-5p in inhibiting ovarian cancer cell proliferation and survival via direct suppression of transcriptional co-activators YAP and TAZ. Oncotarget 6: 8676-8686, 2015.

28. Duan L, Hao X, Liu Z, Zhang Y and Zhang G: miR-129-5p is down-regulated and involved in the growth, apoptosis and migration of medullary thyroid carcinoma cells through targeting RET. FEBS Lett 588: 1644-1651, 2014.

29. Li M, Tian L, Wang L, Yao H, Zhang J, Lu J, Sun Y, Gao X, Xiao H and Liu M: Down-regulation of miR-129-5p inhibits growth and induces apoptosis in laryngeal squamous cell carcinoma by targeting APC. PLoS One 8: e77829, 2013.

30. Huang ZM, Yang J, Shen XY, Zhang XY, Meng FS, Xu JT, Zhang BF and Gao HJ: MicroRNA expression profile in non-cancerous colonic tissue associated with lymph node metastasis of colon cancer. J Dig Dis 10: 188-194, 2009.

31. Hu T, Weng S, Tang W, Xue R, Chen S, Cai G, Cai Y, Shen X, Zhang S and Dong L: Overexpression of BIRC6 is a predictor of prognosis for colorectal cancer. PLoS One 10: e0125281, 2015.

32. Luk SU, Xue H, Cheng H, Lin D, Gout PW, Fazli L, Collins CC, Gleave ME and Wang Y: The BIRC6 gene as a novel target for therapy of prostate cancer: Dual targeting of inhibitors of apoptosis. Oncotarget 5: 6896-6908, 2014.

33. Tang W, Xue R, Weng S, Wu J, Fang Y, Wang Y, Ji L, Hu T, Liu T, Huang X, et al: BIRC6 promotes hepatocellular carcinogenesis: Interaction of BIRC6 with p53 facilitating p53 degradation. Int J Cancer 136: E475-E487, 2015. 